Supporting Information

\title{
Nitrogen Activation on Defective Potassium Chloride and Sodium Chloride
}

\author{
Michael Häfner and Thomas Bredow* \\ Mulliken Center for Theoretical Chemistry, Institut für Physikalische und Theoretische \\ Chemie, Universität Bonn, Beringstraße 4, 53115 Bonn, Germany \\ E-mail: bredow@thch.uni-bonn.de \\ Phone: +49 (0)228 733839. Fax: +49 (0)228 739064
}




\section{Frequency Calculations}

Optimally, frequency calculations are performed including all atoms in a system. While it is safe to assume that most ions of the solid slab model are not involved in the surface reaction and thus does not contribute to the thermal corrections, the ions surrounding the reactive center might still contribute in a significant manner. To assess their influence on the thermal corrections, the zero-point vibrational correction was assessed for four systems and two sets of modes. In one set, only the molecular vibrations were considered, in the other set, the two ion shells surrounding the defect were considered as well. The results of this assessment are shown in table S1.

Table S1: Comparison of the vibrational corrections between molecular vibrations only and including the local surface ions for the first four steps of the 1,1-addition of hydrogen to nitrogen; calculations were done on $\mathrm{KCl}$ using PBED3(BJ) $/ 500 \mathrm{eV}$, energies in $\mathrm{eV}$.

\begin{tabular}{cccc}
\hline system & $\begin{array}{c}\text { molecular only } \\
\mathrm{eV}\end{array}$ & $\begin{array}{c}\text { including local surface } \\
\mathrm{eV}\end{array}$ & $\begin{array}{c}\text { difference } \\
\mathrm{eV}\end{array}$ \\
\hline $\mathrm{N}_{2}$ & 0.003 & 0.014 & 0.011 \\
$\mathrm{~N}_{2}+\mathrm{H}_{2}$ & 0.030 & 0.043 & 0.013 \\
iso- $\mathrm{N}_{2} \mathrm{H}_{2} \mathrm{TS}$ & 0.066 & 0.079 & 0.013 \\
iSO- $\mathrm{N}_{2} \mathrm{H}_{2}$ & 0.273 & 0.291 & 0.018 \\
\hline
\end{tabular}

In no case does the error amount to more than $0.02 \mathrm{eV}$. In addition, the error always carries the same sign and magnitude, varying between 0.01 and $0.02 \mathrm{eV}$. The largest error occurs with the adsorption and the influence on the energies of the actual reaction is even less significant. Therefore, all frequency calculations were carried out with molecular modes only, saving significant computational cost at negligible loss of accuracy. 